\title{
Annular aperture arrays: study in the visible region of the electromagnetic spectrum
}

\author{
J. Salvi, M. Roussey, F. I. Baida, M.-P. Bernal, A. Mussot, T. Sylvestre, H. Maillotte, and D. Van Labeke \\ FEMTO-ST, UMR 6174, Département d'Optique P. M. Duffieux, Université de Franche-Comté, 16 Route de Gray, \\ 25030 Besançon Cedex, France
}

A. Perentes, I. Utke, C. Sandu, and P. Hoffmann

Institut d'Imagerie et Optique Appliquée, École Polytechnique Fédérale de Lausanne, CH-1015 Lausanne, Switzerland

B. Dwir

Institut de Photonique et d'Électronique Quantiques, École Polytechnique Fédérale de Lausanne, CH-1015 Lausanne, Switzerland

Received November 8, 2004

\begin{abstract}
Baida and Van Labeke recently proposed a structure that exhibits a supertransmission of light through an array of nanometric coaxial apertures in a metallic film that has been named an annular aperture array (AAA) [Opt. Commun. 209, 17 (2002); Phys. Rev. B 67, 155314 (2003); J. Microsc. 213, 140 (2003)]. We present the first experimental study, to our knowledge, of an AAA structure in the visible region. For technological reasons, the structure under study does not produce a supertransmission of $80 \%$ as in Baida and Van Labeke [Opt. Commun. 209, 17 (2002)]. We built the nanostructure and experimentally recorded its far-field spectral response. This transmission shows only one broad band with a maximum around $\lambda$ $=700 \mathrm{~nm}$, giving a maximum efficiency around $17 \%$. A finite-difference time-domain simulation reproduces quite well the obtained transmission spectrum. (C) 2005 Optical Society of America

OCIS codes: $050.1220,240.7040,120.2440$.
\end{abstract}

Ebbesen et al. ${ }^{1}$ showed in 1998 that extraordinary light transmission could be achieved through a subwavelength array of cylindrical holes in metallic films. Following this work, Baida and Van Labeke recently proposed a structure that could exhibit a higher transmission. ${ }^{2-4}$ This structure consists of an array of subwavelength coaxial apertures in a real metal (gold, for instance), and it has been named an annular aperture array (AAA). The coaxial cavities offer the advantage of showing a guided mode with a cutoff wavelength in the red region of the spectrum contrary to a simple cylindrical waveguide in the same metal. ${ }^{5}$ This kind of nanostructure could have interesting applications (filters, flat displays, nanolithography, etc.). Finite-difference time-domain (FDTD) simulations have established that a $90 \%$ transmission could be reached in the visible region of the spectrum. ${ }^{3}$ However, the designed structure has several difficult technical constraints. First, the metal is silver, which is not well adapted to nanofabrication. Second, the period and the coaxial radii must be very small (period $300 \mathrm{~nm}$, inner radius $50 \mathrm{~nm}$, and outer radius $75 \mathrm{~nm}$ ). Recently, 80\% transmission through an AAA structure was experimentally established in the near-infrared part of the spectrum $(\lambda=1.97 \mu \mathrm{m}){ }^{6}$

We plan to build an AAA structure to have a large transmission in the visible region, and we present here our first experimental results. So far, the AAA structure has been fabricated in gold by electron lithography and gold lift-off. We present images of the fabricated structure by electronic microscopy and atomic force microscopy (AFM). We experimentally record the structure's far-field spectral response by using a bright fibered light source that presents a continuum spectrum in the visible range.

The numerical simulation uses a homemade FDTD code already described elsewhere..$^{24}$ The $3 \mathrm{D}$ calculations use Berenger's perfectly matched layers (PMLs) boundary conditions to cancel the parasitic reflections on the edges of the calculation window. The latter technique treats only the homogeneous waves. ${ }^{7}$ Therefore, to avoid unwanted reflections caused by evanescent waves, the object is placed at a distance from the edges larger than half of the highest value of the illuminating wavelength. In addition, to obtain accurate results, the spatial meshing of the object must be small, which implies a great number of spatial nodes. To decrease the number of nodes, we use a nonuniform spatial discretization of the metallic structure as described in Ref. 8.

In the present study, the object consists of a biperiodic (in the $x$ and $y$ directions) array of coaxial gold apertures. The metallic layer is deposited on a glass substrate that is also incorporated into our simulations. The calculation window consists of one array period (see Fig. 1, where four periods are presented).

The absorbing PMLs are therefore necessary only in the $z$ direction. Periodic conditions are applied in the $x$ and $y$ directions. The spatial increment step is set to $\Delta_{x}=\Delta_{y}=10 \mathrm{~nm}$. The nonuniform meshing is done in the $z$ direction. A spatial step of $\delta_{z}=10 \mathrm{~nm}$ is also chosen to mesh the metallic film, whereas $\Delta_{z}$ $=25 \mathrm{~nm}$ for the rest of the window. This mesh variation must be carried out gradually to avoid artificial reflections. ${ }^{9}$ 


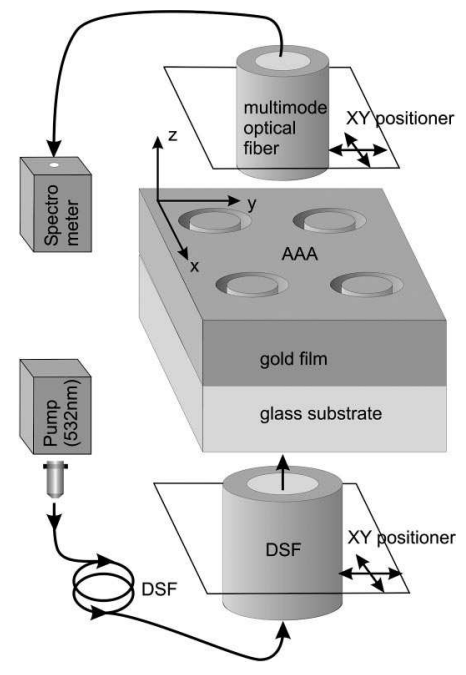

Fig. 1. Scheme of the studied structure and experimental setup.

For the sample under study, the periodicity of the coaxial apertures is $p=600 \mathrm{~nm}$ and the gold-layer thickness is $h=150 \mathrm{~nm}$. The incident field is a pulsed plane wave polarized in the $x$ direction and propagating along the $z$ axis (normal incidence from the glass substrate). It is centered on $\lambda_{c}=650 \mathrm{~nm}$, and it covers the spectral range between 530 and $900 \mathrm{~nm}$. For real metal, like gold, silver, or aluminum, the dispersion of the dielectric permittivity must be taken into account. By use of Drude's analytical model, a simple computing scheme connecting the displacement vector to the electric field is obtained. It can be easily incorporated into the usual leap-frog FDTD scheme. ${ }^{10}$ For this work, we studied a broad spectral range, and therefore the dispersion of gold cannot be described by only one simple Drude's model. To bypass this problem, the spectral range is subdivided into small spectral intervals of $\delta \lambda=10 \mathrm{~nm}$. In every spectral domain, the tabulated experimental permittivities ${ }^{11}$ are fit with an appropriate Drude's model and a FDTD calculation is performed.

The transmission is obtained by evaluating the zero-order diffracted intensity normalized by the incident intensity. In fact, because of the periodicity of the structure $(p=600 \mathrm{~nm})$, only one diffracted order (zero order) exists for wavelengths larger than $p$. For wavelengths between $\lambda=532 \mathrm{~nm}$ (beginning of our continuum) and $\lambda=600 \mathrm{~nm}$, higher diffracted orders appear $( \pm 1)$, but they are not experimentally recorded because they propagate far from the fiber axis (see below).

The 2D arrays of coaxial apertures are generated by electron-beam lithography and gold lift-off. The gold structures are made on a $20 \mathrm{~nm}$ thick solgelcoated transparent conductive oxide layer on a microscope slide. The resist is spin coated to $700 \mathrm{~nm}$ thickness to obtain the necessary high-aspect-ratio resist structures. The electron lithography equipment is based on a JEOL 6400 JSM scanning electron microscope (SEM) with a $\mathrm{LaB}_{6}$ thermionic emitter. The electron beam is controlled with Nabity Systems patterning software (NPGS). The exposure dose is varied between 200 and $250 \mu \mathrm{C} / \mathrm{cm}^{2}$. With $250 \mu \mathrm{C} / \mathrm{cm}^{2}$ the structures obtained correspond best to the calculated geometry (see Fig. 2).

After developing in the surfactant containing the developer, oxygen plasma treatment, and adhesionpromoting titanium evaporation coating ( $5 \mathrm{~nm}$ thick), $150 \mathrm{~nm}$ of gold is deposed by electron-beam evaporation. Finally, gold lift-off is achieved in an ultrasonic acetone bath.

SEM and AFM images of one of the best-looking arrays are presented in Fig. 2. The images consist of a large array of coaxial apertures with inner and outer diameters of 250 and $330 \mathrm{~nm}$, respectively, and a grating period of $p=600 \mathrm{~nm}$. Note that the apertures are well aligned and that the AAA does not present any important defects. The AFM line scan does not reveal the full depth of the cylindrical holes because of the cone angle of the AFM tip.

The experimental setup is shown in Fig. 1. A local illumination, under normal incidence, is obtained by use of a powerful white-light fiber source. ${ }^{12}$ The laser output beam is focused into a $100 \mathrm{~m}$ dispersionshifted fiber (DSF) generating a broadband singlemode supercontinuum by stimulated Raman scattering and parametric four-wave mixing. To collect the light transmitted through the AAA, a cleaved-end multimode fiber $(62.5 \mu \mathrm{m}$ core diameter) is brought to around $20 \mu \mathrm{m}$ close to the sample. The spectrum is then recorded by means of an optical spectrum analyser (S2000 Miniature Fiber Optic Spectrometer from Ocean Optics).

Figure 3 shows an image of a zone of the sample. It consists of a large area containing four AAAs and one triangular metal-free test zone. The image is obtained by means of a classical optical microscope used in transmission with white light. The color modification reflects the variations of the spectral properties of AAA structures when changing its geometric parameters. To obtain the spectral response of one matrix of AAA (see Fig. 2), we have to record the source spectrum through the metal-free test zone.

Figure 4 shows the experimental spectral response of an AAA zone presented in Fig. 2 compared to the theoretical spectral response obtained by the FDTD calculation. We would like to point out that, apart from a background subtraction, no image treatment was performed on the curve. The noise appearing in the experimental spectrum is probably induced by temporal instability of the laser and mechanical instabilities in the injection and/or detection processes.

One can see from Fig. 4 that there is good agreement between experience and theory. For both curves
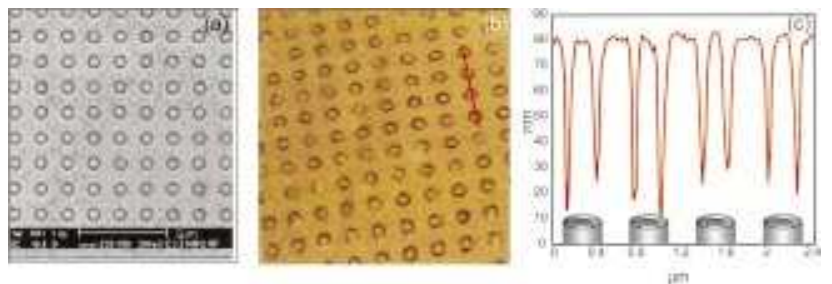

Fig. 2. (a) SEM and (b) AFM $(10 \mu \mathrm{m} \times 10 \mu \mathrm{m})$ images of the AAA under study. (c) Cross section (in red) made over four annular apertures shown by the red line in (b). 


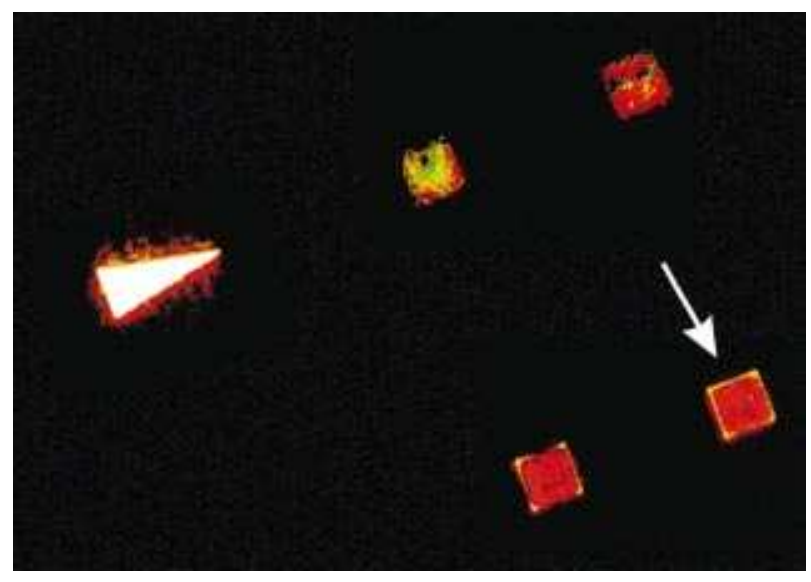

Fig. 3. Far-field image of one sample obtained with a conventional microscope. The white arrow indicates the AAA for which the spectral response has been studied.

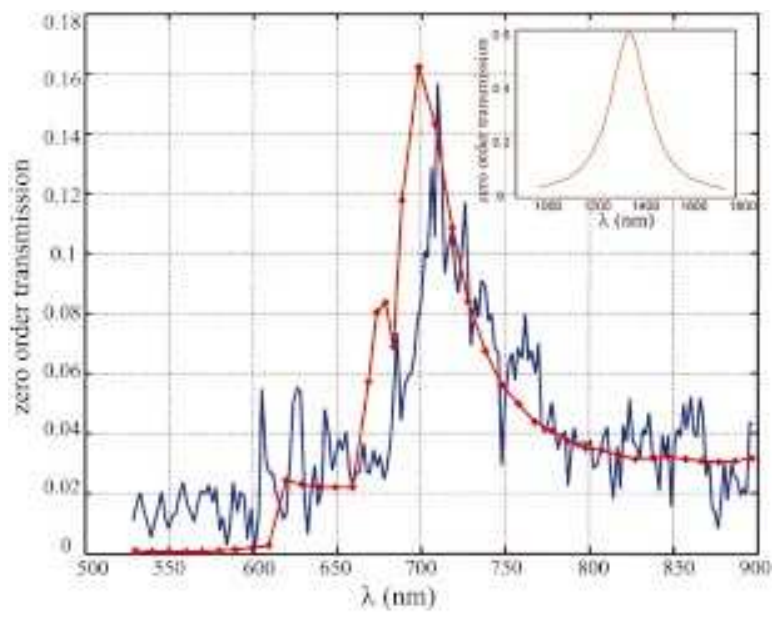

Fig. 4. Theoretical (red) and experimental (blue) spectral responses of the studied AAA structure located by the white arrow in Fig. 3. Inset, theoretical transmission in the nearinfrared region showing a maximum of transmission at $\lambda$ $=1330 \mathrm{~nm}$.

a transmission peak around $700 \mathrm{~nm}$ (theoretically around 16\%) is observed. The experimental curve is slightly translated toward higher wavelengths. This shift is probably linked to the polarization direction of the incident beam (see Fig. 7 in Ref. 2). In the theoretical simulation, the polarization was set along the $x$ or $y$ direction. The experimental setup could not allow us to control this parameter. Moreover, the fabrication parameters slightly differ from one coax with respect to the other and from the theoretical ones. Finally, the calculation does not take into account the very thin titanium layer ( $5 \mathrm{~nm}$ thick) that is used to improve the adhesion of gold onto the substrate.
In this Letter we have presented the first study, to our knowledge, of transmission through an AAA structure in the visible region of the spectrum. A FDTD calculation reproduces quite well the experimental spectrum. Nevertheless, in this first attempt, the physical parameters of the structure only lead to a maximum of transmission around $17 \%$ for $\lambda=700 \mathrm{~nm}$. In fact, more than $60 \%$ of transmission for the fabricated structure is theoretically found at $\lambda=1330 \mathrm{~nm}$ (see the inset in Fig. 4). The peak at $\lambda=700 \mathrm{~nm}$ is then the first harmonic of the $\mathrm{TE}_{11}$ mode as demonstrated in Ref. 5 .

The fabrication of other structures is in progress at the École Polytechnique Fédérale de Lausanne. The challenge is difficult as it needs a strong enhancement of our technological process to reduce all the dimensions (period and radii) by a factor of 2 . Moreover, the theoretical study of the transmission of light by AAA structures remains an interesting subject. For instance, the transmission spectrum obtained in this Letter is different from those published in Refs. 2 and 3. This difference has to be studied in more detail and explained in order to design new structures and to optimize their parameters.

This research was supported by the Laboratoire Européen Associé en Microtechnique Franco-Suisse, and we also thank the Swiss National Science Foundation (N 200021-103333) for partial financial support of this work. F. I. Baida's e-mail address is fbaida@univ-fcomte.fr.

\section{References}

1. T. Ebbesen, H. Lezec, H. Ghaemi, T. Thio, and P. Wolff, Nature 391, 667 (1998).

2. F. I. Baida and D. V. Labeke, Opt. Commun. 209, 17 (2002).

3. F. I. Baida and D. V. Labeke, Phys. Rev. B 67, 155314 (2003).

4. D. V. Labeke, F. I. Baida, and J. M. Vigoureux, J. Microsc. 213, 140 (2003).

5. F. I. Baida, D. V. Labeke, G. Granet, A. Morcau, and A. Belkir, Appl. Phys. B 79, 1 (2004).

6. W. Fan, S. Zhang, B. Minhas, K. J. Malloy, and S. R. J. Bruech, Phys. Rev. Lett. 94, 033902 (2005).

7. J. P. Berenger, J. Comput. Phys. 114, 185 (1994).

8. J. Seidel, F. I. Baida, L. Bischoff, B. Guizal, S. Grafstrom, D. V. Labeke, and L. M. Eng, Phys. Rev. B 69, 121405 (2004).

9. A. Taflove and S. C. Hagness, Computational Electrodynamics: the Finite-Difference Time-Domain Method, 2nd ed. (Artech House, 2000).

10. F. I. Baida, D. V. Labeke, and Y. Pagani, Opt. Commun. 255, 241 (2003).

11. P. B. Johnson and R. W. Christy, Phys. Rev. B 6, 4370 (1972).

12. A. Mussot, T. Sylvestre, L. Provino, and H. Maillotte, Opt. Lett. 28, 1820 (2003). 